\title{
Effects of Spatially Uncertain Structural Properties on Helicopter Aeroelastic Response Predictions using High Dimensional Model Representation
}

\author{
Senthil Murugan ${ }^{*}$ Rajib Chowdhury † Sondipon Adhikari $\ddagger$ and Michael I. Friswell ${ }^{\ddagger}$ \\ Swansea University, UK
}

\begin{abstract}
This paper studies the effects of spatially uncertain material properties of composite helicopter rotors on vibratory hub load predictions. The flap, lag and torsional stiffnesses of the rotor blades are considered to vary spatially along the axis of the rotor blades. The spatial uncertainty analysis is performed with rotors modeled as both isotropic rotors and dissimilar rotor systems. In isotropic rotor systems, the uncertainty is considered to be similar for all of the four blades and the uncertainty effects on the $4 /$ rev vibratory hub loads are studied. To reduce the computational expense of uncertainty analysis, a high dimensional model representation (HDMR) method is developed to approximate the vibratory loads as functions of blade stiffness properties modeled as random fields. In the HDMR formulation, a Karhunen-Loève expansion and a low order expansion are used to represent the input and outputs, respectively. Monte Carlo simulations are then performed with the developed HDMR models. The proposed approach decouples the computationally expensive aeroelastic simulations of uncertainty analysis. MCS, performed with computationally less expensive HDMR models, shows that spatial uncertainty has considerable influence on the $4 / \mathrm{rev}$ vibratory hub loads. In the uncertainty analysis of dissimilar rotor systems, the spatial uncertainty of each blade of the rotor is assumed to be independent of the other blades. Spatial uncertainties of the rotor with dissimilar blades have considerable influence on the $1 / \mathrm{rev}$ and $2 / \mathrm{rev}$ vibratory hub loads in addition to the $4 / \mathrm{rev}$ vibratory hub loads. The $1 / \mathrm{rev}$ and $2 / \mathrm{rev}$ harmonics of longitudinal and lateral vibratory hub forces, and $1 /$ rev of vertical vibratory hub force and all three vibratory hub moments are highly sensitive to spatially uncertain material properties.
\end{abstract}

\section{Introduction}

$\mathrm{T}$ he quantification of uncertainty in aeroelastic response prediction enhances the reliable and robust design of aerospace vehicles and can considerably reduce the cost of flight test experiments. ${ }^{1-8}$ A recent review on uncertainty quantification in aeroelasticty is given by Pettit. ${ }^{1}$ Structural, aerodynamic and control parameters used in the aeroelastic design process are uncertain in nature due to manufacturing defects or service time degradation. From the structural perspective, the material properties of composites which are extensively used in aircraft structures are uncertain. Uncertainty in composite effective material properties occur because of variations associated with the fiber and matrix material properties, fiber volume ratio, ply orientation, fiber waviness or undulation, voids, incomplete curing of resin, excess resin between plies, and variation in ply thickness. ${ }^{9,} 10$ Thus the consideration of randomness in composite material properties is very important for reliable aeroelastic predictions. Helicopter rotor blades, which play a dominant role in the overall vehicle performance, are typically made of composites and involve complex manufacturing processes. Further, the helicopter rotor system operates in a highly unsteady aerodynamic environment leading to severe vibratory loads. ${ }^{11}$ Repeated exposure to this severe loading condition can deteriorate the structural

\footnotetext{
${ }^{*}$ Research Assistant, College of Engineering, Swansea University, UK, Email: s.m.masanam@swansea.ac.uk, AIAA Senior Member.

${ }^{\dagger}$ Newton Fellow, College of Engineering, Swansea University, UK.

${ }^{\ddagger}$ Professor of Aerospace Engineering, College of Engineering, Swansea University, UK, Senior Member AIAA

$\S$ Professor of Aerospace Structures, College of Engineering, Swansea University, UK.
} 
or material characteristics of composite rotor blades. Therefore, the effect of uncertain material properties of composite rotor blades on the aeroelastic response has to be quantified.

Murugan et al. ${ }^{12-15}$ studied the effects of uncertain material properties on the aeroelastic response predictions of composite helicopter rotors. The aeroelastic response of helicopter rotor blades has shown significant scatter from the baseline values due to material uncertainty. You et al. ${ }^{16}$ studied the influence of uncertain material properties and fabrication/ manufacturing uncertainties modeled as random variables on the aeroelastic response of composite rotor blades. Murugan et al. ${ }^{12,13,15}$ assumed the effects of randomness in material properties to be uniform throughout the span of the rotor blade. However, composite material properties can vary considerably along the span of the blade. ${ }^{3,9}$ Sriramula and Chryssanthopoulos ${ }^{9}$ reviewed studies that considered effects of spatial variability in vibration, buckling and reliability problems of composite structures. Therefore, it is important to study the effects of spatially uncertain material properties on the aeroelastic response predictions of rotor blade. Uncertainty analysis of helicopter can be studied with rotors modeled as an isotropic rotor or as a dissimilar rotor. In an isotropic rotor system, all the blades are considered to have similar structural properties whereas in a dissimilar rotor, each or multiple blades are assumed to have properties different from other blades.

Aeroelastic uncertainty analyses are, generally, performed with direct Monte Carlo simulations which are computationally expensive. ${ }^{12,13,17}$ Response surface methods and polynomial chaos expansion are used to reduce the computational cost of uncertainty analysis with parametric uncertainties modeled as random variables. $^{5,8,17}$ However, there are very few studies focussed on developing a non-intrusive uncertainty analysis method to study the effects of spatial uncertainties on the aeroelastic response. In this study, the Karhunen-Loève (K-L) expansion ${ }^{18}$ is used to discretize the input random field and high dimensional model representation (HDMR $)^{19}$ is used to approximate the output response in terms of functions of lower dimensions as an efficient uncertainty propagation model. The present approach has several advantages. First is the reduction in data processing. The exponentially growing number of function evaluations is represented via polynomially growing tables ${ }^{20,21}$ for each function component (terms in the general expansion). This property improves the computational efficiency for high dimensional problems. The second advantage is the reduction of computational complexity. HDMR is generated by a family of projection operators. ${ }^{19}$ Consequently, the method splits any problem into easier low-dimensional subproblems. The third advantage is that HDMR is a mean square convergent series expansion, because of the inherent orthogonal properties of HDMR component functions. ${ }^{19}$

The HDMR approach discussed above decouples the computationally expensive aeroelastic simulations and the stochastic analysis. The effects of spatial variation in the stiffness properties of rotor blade are studied with HDMR based MCS. Uncertainty analysis is performed with rotors modeled as isotropic rotor systems and dissimilar rotor systems. In the isotropic rotor system, spatial uncertainty analysis is performed with direct MCS and MCS based on HDMR models. Numerical results from the HDMR models are compared with direct MCS and the effects of spatial uncertainty on vibratory hub loads are studied. In the dissimilar rotor system, uncertainty effects are studied with MCS. The helicopter aeroelastic model and HDMR models used in this study are discussed in the following sections.

\section{Helicopter Aeroelastic Model}

A comprehensive aeroelastic analysis code, UMARC, based on the finite element method, is used to evaluate the helicopter blade response. ${ }^{22}$ UMARC has been extensively used by industry and academia for helicopter aeroelastic response predictions. ${ }^{23,24}$ The aeroelastic formulation is briefly given in the following. The helicopter is modeled as a non-linear representation of composite rotor blades coupled to a rigid fuselage with 6-degrees of freedom. The rotor blade is modeled as a slender elastic beam undergoing flap bending displacement $(w)$, lag bending displacement $(v)$, elastic twist $(\phi)$, and axial deflection $(u)$ with a rotational speed of $\Omega$ as shown in Fig. 1(a). The effect of moderate deflections is included by retaining second order non-linear terms. For a given blade, the governing equations are derived using a generalized Hamilton's principle applicable to non-conservative system as

$$
\int_{\psi_{1}}^{\psi_{2}}(\delta U-\delta T-\delta W) d \psi=0
$$

Here, $\delta U$ is the virtual strain energy and $\delta T$ is the virtual kinetic energy of the elastic blade. Also, $\delta W$ is the virtual work done by the external aerodynamic forces acting on the blade and $\psi=\Omega t$ is the azimuth 
angle of the blade around the rotor disk. The Leishman-Beddoes unsteady aerodynamic model with the Bagai and Leishman free wake model is used for aerodynamic load calculations. ${ }^{22}$ The blade is discretized into beam finite elements, each with fifteen degrees of freedom. These degrees of freedom correspond to cubic variations in axial and bending (flap and lag) deflections, and quadratic variations in elastic torsion. The finite element equations are reduced in size by using a normal mode transformation. This results in the system of non-linear ordinary differential equation with periodic coefficients given below.

$$
\mathbf{M} \ddot{\mathbf{p}}(\psi)+\mathbf{C}(\psi) \dot{\mathbf{p}}(\psi)+\mathbf{K}(\psi) \mathbf{p}(\psi)=\mathbf{F}(\mathbf{p}, \dot{\mathbf{p}}, \psi)
$$

Here $\mathbf{M}, \mathbf{C}, \mathbf{K}, \mathbf{F}$, and $\mathbf{p}$ represent the finite element mass matrix, damping matrix, structural stiffness matrix, finite element force vector, and modal displacement vector, respectively. Non-linearities in the model occur primarily due to Coriolis terms and due to moderate deflections in the strain-displacement relations. Equation (2) governs the dynamics of the rotor blade. These equations are then solved using the finite element in time method in combination with the Newton-Raphson method. The solution to these equations are then used to calculate rotor blade loads using the force summation method where aerodynamic forces are added to the inertial forces. The blade loads are integrated over the blade length and transformed to the fixed frame to estimate hub loads. The steady hub loads are then used to obtain the forces acting on the rotor and combined with fuselage and tail rotor forces to obtain the helicopter rotor trim equations:

$$
\mathcal{F}_{\mathcal{T}}\left(\Theta_{T}\right)=\mathbf{0}
$$

Here, the symbol $\Theta_{T}$ represents the flight control angles evaluated in the trim process. ${ }^{11}$ These non-linear trim equations are also solved using the Newton-Raphson method. The helicopter rotor trim equations and the blade response equations in (2) and (3) are solved simultaneously to obtain the blade steady response and hub loads. This coupled solution procedure is important to capture the aeroelastic interaction between the aerodynamic forces and the blade deformations. Further details of the aeroelastic analysis are available in the UMARC theory manual. ${ }^{22}$

\section{A. Isotropic and Dissimilar Rotor System}

The aeroelastic analysis discussed above varies for the isotropic rotor system and the dissimilar rotor system. In an isotropic rotor system, the harmonics of vibratory loads $\left(n N_{b} /\right.$ rev, where $n$ is an integer) that are multiples of the number of blades $\left(N_{b}\right)$ are transmitted to the fuselage. In a dissimilar rotor system, all the $n /$ rev harmonics of vibratory loads are transmitted to the fuselage. For a dissimilar rotor, the steady and vibratory components of blade loads of each blade are calculated individually using the force summation method. ${ }^{25}$ The fixed-frame hub loads are then obtained by summing the contributions from each blade and the trim analysis is performed as discussed above.

\section{Random Field Discretization}

The representation and discretization of spatial uncertainty as a random field is discussed in this section. A random field over $\varepsilon$ is formally defined as a indexed set of random variables $\left\{\kappa_{x}(\theta)\right\}_{x \in \Omega}$, where $\kappa_{x}(\theta)$ are random variables with values in $\varepsilon$, defined on a probability space $(\Theta, \mathcal{B}, P)$. It should be noted that a probability space consists of three parts: (i) a sample space, $\Theta$, which is the set of all possible outcomes; (ii) a set of events, where each event is a set containing zero or more outcomes (the collection of all such events is a $\sigma$-algebra $\mathcal{B}$ ); and (iii) the assignment of probabilities to the events, that is, a function from events to probability levels, using the probability measure function, $P . \Omega$ can be a finite or countable set, in which case the stochastic process is called a discrete stochastic process, or an uncountable set such as an interval $\Omega \subset \mathbb{R}$ or even a domain $\Omega \subset \mathbb{R}^{d}$. In the case where $\Omega$ is a spatial domain, the random process is called a random field. A stochastic process can be equivalently seen as a measurable function

$$
\kappa:(x, \theta) \in \Omega \times \Theta \mapsto \kappa(x, \theta) \in \varepsilon
$$

or alternatively as a random variable with values in a space of functions defined on $\Omega$ with values in $\varepsilon$. The equivalence between these different interpretations requires some technical considerations. In the following, we restricts the presentation to scalar random processes, i.e. $\varepsilon=\mathbb{R}$.

The characterization of a random process requires the probabilistic characterization of a set of random variables, eventually uncountable. A random process can be completely characterized by its finite 


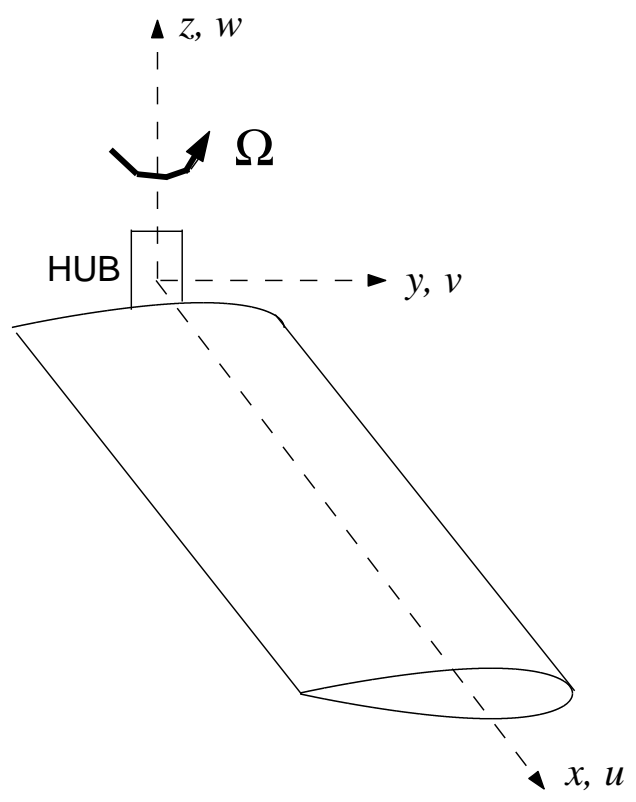

(a) Elastic rotor blade.

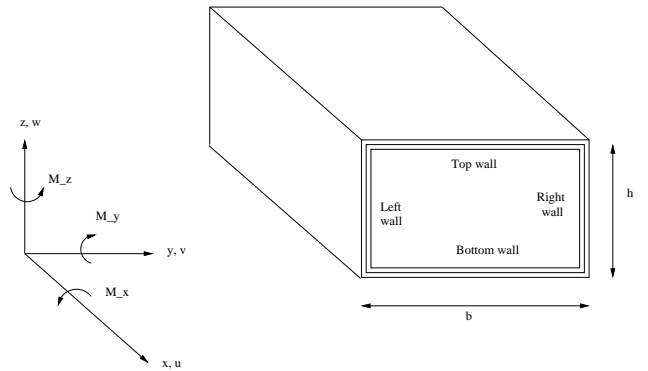

(b) Composite box beam.

Figure 1. Composite helicopter rotor blade.

dimensional probability laws, which are the joint probability laws of all finite sets of random variables $\left\{\kappa_{x_{1}}(\theta), \ldots, \kappa_{x_{n}}(\theta)\right\}, n \in \mathbb{N}, x_{i} \in \Omega$. Moreover, the random process is homogeneous if its mean $\mu(x, \theta)$ and variance $\sigma^{2}(x, \theta)$ are constant and its autocorrelation coefficient $\rho\left(x, x^{\prime}\right)$ is a function of $x-x^{\prime}$ only. This autocorrelation function may take many distinct forms. One such form is the exponential type,

$$
\rho\left(x, x^{\prime}\right)=e^{-\alpha_{1}\left|x_{(1)}-x^{\prime}{ }_{(1)}\right|-\alpha_{2}\left|x_{(2)}-x^{\prime}{ }_{(2)}\right|}
$$

where $x_{(i)}$ denotes the $i^{\text {th }}$ coordinate of $x$ and $\alpha_{1}, \alpha_{2}$ are known as correlation lengths.

In the following, we consider the representation and discretization of second-order processes. A wide variety of discretization schemes are available in the literature. ${ }^{26-28}$ The Karhunen-Loève (K-L) decomposition which is classically used in the context of spectral stochastic methods is discussed here. K-L decomposition $^{28}$ applies to second order stochastic processes $\kappa \in L^{2}(\Omega) \otimes L^{2}(\Theta, d P)$. The random process $\kappa(x, \theta)$ is decomposed in the form

$$
\kappa(x, \theta)=\bar{\kappa}(x)+\sum_{i=1}^{\infty} \sqrt{\lambda_{i}} \psi_{i}(x) \zeta_{i}(\theta)
$$

where $\bar{\kappa}(x)$ is the mean value of $\kappa(x, \theta)$, the functions $\psi_{i}(x)$ form a particular Hilbertian basis of $L^{2}(\Omega)$, $\zeta_{i} \in L^{2}(\Theta, d P)$ are centered uncorrelated random variables with unit variance and $\lambda_{i}$ are positive constants. Couples $\left(\psi_{i}, \lambda_{i}\right) \in L^{2}(\Omega) \times \mathbb{R}^{+}$are solutions of the eigenproblem

$$
\int_{\Omega} \Gamma_{\kappa}\left(x, x^{\prime}\right) \psi_{i}\left(x^{\prime}\right) d x^{\prime}=\lambda_{i} \psi_{i}(x)
$$

where $\Gamma_{\kappa}$ is the covariance function of $\kappa(x, \theta)$, defined by

$$
\Gamma_{\kappa}(x, y)=E\left[(\kappa(x, \theta)-\bar{\kappa}(x))\left(\kappa\left(x^{\prime}, \theta\right)-\bar{\kappa}\left(x^{\prime}\right)\right)\right]
$$

In practice, the infinite series in Eq. (6) must be truncated, yielding a truncated K-L approximation

$$
\tilde{\kappa}(x, \theta)=\bar{\kappa}(x)+\sum_{i=1}^{P} \sqrt{\lambda_{i}} \psi_{i}(x) \zeta_{i}(\theta)
$$


which approaches $\kappa(x, \theta)$ in the mean square sense as the positive integer $P \rightarrow \infty$. For linear or exponential covariance functions and simple domains, the eigensolutions can be evaluated analytically. According to Eq. (9), the K-L approximation provides a parametric representation of $\kappa(x, \theta)$ and, hence, of $\tilde{\kappa}(x, \theta)$ with $P$ random variables. The K-L expansion has uniqueness and error-minimization properties that make it a convenient choice over other available methods. Generally speaking, if $\zeta_{i}(\theta)$ contains non-Gaussian random variables, then it is difficult to obtain a representation in independent random variables, as un-correlation and independence are not equivalent. Obtaining a representation in independent random variables for a non-Gaussian field $\kappa(x, \theta)$ usually involves nonlinear transformations of the random variables. ${ }^{29-31}$

\section{High Dimensional Model Representation}

The High Dimensional Model Representation of an arbitrary $M$-dimensional response $f(\mathbf{x}), \mathbf{x} \in \mathfrak{R}^{M}$ can be derived by partitioning the identity operator $\mathcal{I}$, called $\mathcal{I}_{M}$ in the M-dimensional case and also in the 1D case hereafter, with respect to the projectors $\mathcal{P}_{1}, \mathcal{P}_{2}, \ldots, \mathcal{P}_{M}$. This can be expressed as ${ }^{19}$

$$
\begin{aligned}
\mathcal{I}_{M} & =\prod_{m=1}^{M}\left(\mathcal{P}_{m}+\left(\mathcal{I}_{1}-\mathcal{P}_{m}\right)\right) \\
& =\underbrace{\prod_{m=1}^{M} \mathcal{P}_{m}}_{1 \text { term }}+\underbrace{\sum_{m=1}^{M}\left(\mathcal{I}_{1}-\mathcal{P}_{m}\right) \prod_{s \neq m} \mathcal{P}_{s}}_{\left(\begin{array}{c}
M \\
1
\end{array}\right) \text { terms }}+\underbrace{\sum_{m=1}^{M} \sum_{s=m+1}^{M}\left(\mathcal{I}_{1}-\mathcal{P}_{m}\right)\left(\mathcal{I}_{1}-\mathcal{P}_{s}\right) \prod_{p \neq m, s} \mathcal{P}_{p}}_{\left(\begin{array}{c}
M \\
2
\end{array}\right) \text { terms }} \\
& +\cdots+\underbrace{\sum_{m=1}^{M} \mathcal{P}_{m} \prod_{s \neq m}\left(\mathcal{I}_{1}-\mathcal{P}_{s}\right)}_{\left(\begin{array}{c}
M \\
M-1
\end{array}\right) \text { terms }}+\underbrace{\prod_{m=1}^{M}\left(\mathcal{I}_{1}-\mathcal{P}_{m}\right)}_{1 \text { term }}
\end{aligned}
$$

which is composed of $2^{M}$ mutually orthogonal terms and $\mathcal{P}_{m}\left(\mathcal{I}_{1}-\mathcal{P}_{m}\right)=0$. The orthogonal representation of Eq. 10 is a manifestation of the HDMR and can be rewritten as, ${ }^{32,33}$

$$
f(\mathbf{x})=f_{0}+\sum_{i=1}^{M} f_{i}\left(x_{i}\right)+\sum_{1 \leq i<j \leq M} f_{i j}\left(x_{i}, x_{j}\right)+\ldots+f_{123 \ldots M}\left(x_{i}, x_{2}, \ldots, x_{M}\right)=\sum_{l=0}^{M} \eta_{l}(\mathbf{x})
$$

where $f_{0}$ is a constant term representing the zeroth-order component function or the mean response of output function $f(\mathbf{x}) . f_{i}$ is the first-order term expressing the effect of variable $x_{i}$ acting alone upon the output $f(\mathbf{x})$, and this function is generally nonlinear. The function $f_{i j}\left(x_{i}, x_{j}\right)$ is a second-order term which describes the coupled effects of the variables $x_{i}$ and $x_{j}$ upon the response. The higher order terms gives the coupled effects of increasing numbers of input variables on the output. The last term $f_{123 \ldots M}\left(x_{i}, x_{2}, \ldots, x_{M}\right)$ contains any residual dependence of all the input variables locked together in a cooperative way to influence the output. Once all of the relevant component functions in Eq. (11) are determined and suitably represented, then the component functions constitute the HDMR, thereby replacing the original computationally expensive method of calculating the response by the computationally efficient meta model.

To generate the HDMR approximation of any function, more precisely the cut-center based HDMR, first a reference point $\overline{\mathbf{x}}=\left(\bar{x}_{1}, \bar{x}_{2}, \ldots, \bar{x}_{M}\right)$ has to be defined in the variable space. In the convergence limit, where all correlated functions in Eq. (11) are considered, the cut-HDMR is invariant to the choice of reference point $\overline{\mathbf{x}}$. However in practice the choice of reference point $\overline{\mathbf{x}}$ is important for the cut-HDMR, especially if only the first few terms, say up to first- and second-order, in Eq. (11) are considered. Sobol ${ }^{34}$ showed that the reference point $\overline{\mathbf{x}}$ at the middle of the input domain appears to be the optimal choice. The expansion functions are determined by evaluating the input-output responses of the system relative to the defined reference point in the input variable space. This process reduces to the following relationship for the 
component functions in Eq. (11):

$$
\begin{aligned}
f_{0} & =\int d x f(\mathbf{x}) \\
f_{i}\left(x_{i}\right) & =\int d x^{i} f(\mathbf{x})-f_{0} \\
f_{i j}\left(x_{i}, x_{j}\right) & =\int d x^{i j} f(\mathbf{x})-f_{i}\left(x_{i}\right)-f_{j}\left(x_{j}\right)-f_{0}
\end{aligned}
$$

where, $\int d x^{i}$ means to integrate over all $M$ variables except $x_{i}$ and $\int d x^{i j}$ means to integrate over all $M$ variables except $x_{i}$ and $x_{j}$, etc. These integrals are generally evaluated using numerical integration techniques. Substituting the component functions defined in Eq. (12) into Eq. (11), the general expression of the HDMR can be expressed as

$$
\begin{array}{r}
f(\mathbf{x})=\sum_{1 \leq i_{1}<\ldots<i_{\beta} \leq M} f\left(x_{i_{1}}, \ldots, x_{i_{\beta}} ; \bar{x}^{i_{1}, \ldots, i_{\beta}}\right)-(M-\beta) \sum_{1 \leq i_{1}<\ldots<i_{\beta-1} \leq M} f\left(x_{i_{1}}, \ldots, x_{i_{\beta-1}} ; \bar{x}^{i_{1}, \ldots, i_{\beta-1}}\right) \\
+\frac{(M-\beta+1) !}{2 !(M-\beta-1) !} \sum_{1 \leq i_{1}<\ldots<i_{\beta-2} \leq M} f\left(x_{i_{1}}, \ldots, x_{i_{\beta-2}} ; \bar{x}^{i_{1}, \ldots, i_{\beta-2}}\right)- \\
\ldots \mp \frac{(M-2) !}{(\beta-1) !(M-\beta-1) !} \sum_{1 \leq i \leq M} f\left(x_{i} ; \bar{x}^{i}\right) \pm \frac{(M-1) !}{\beta !(M-\beta-1) !} f(\overline{\mathbf{x}})
\end{array}
$$

where $\beta$ is the order of the HDMR approximation, $1 \leq \beta \leq(M-1)$ and the + or - sign of the last term in Eq. (13) corresponds to $\beta$ being even or odd, respectively. Considering the weak role of the higher-order correlation effects, the approximation is likely to converge at a lower HDMR order, say, $\beta \ll M$. The particular form of Eq. (13) for $\beta=1,2$, or 3 corresponds to first-, second- or third-order HDMR can be explicitly given as

$$
\begin{gathered}
\hat{f}(\mathbf{x})=\sum_{1 \leq i \leq M} f\left(x_{i} ; \bar{x}^{i}\right)-(M-1) f(\overline{\mathbf{x}}), \quad \beta=1 \\
\hat{f}(\mathbf{x})=\sum_{1 \leq i<j \leq M} f\left(x_{i}, x_{j} ; \bar{x}^{i, j}\right)-(M-2) \sum_{1 \leq i \leq M} f\left(x_{i} ; \bar{x}^{i}\right)+\frac{(M-1) !}{2 !(M-3) !} f(\overline{\mathbf{x}}), \quad \beta=2 \\
\hat{f}(\mathbf{x})=\sum_{1 \leq i<j<k \leq M} f\left(x_{i}, x_{j}, x_{k} ; \bar{x}^{i, j, k}\right)-(M-3) \sum_{1 \leq i<j \leq M} f\left(x_{i}, x_{j} ; \bar{x}^{i, j}\right) \\
+\frac{(M-2) !}{2 !(M-4) !} \sum_{1 \leq i \leq M} f\left(x_{i} ; \bar{x}^{i}\right)+\frac{(M-1) !}{3 !(M-4) !} f(\overline{\mathbf{x}}), \quad \beta=3
\end{gathered}
$$

The term $f\left(x_{i} ; \bar{x}^{i}\right)$ is a function of the single $x_{i}$ component (i.e., a cut along $x_{i}$ through the reference point in the function space), while the other variables, $x_{j} \equiv \bar{x}_{j}, j \neq i$, are fixed at the reference point. In the same manner, $f\left(x_{i}, x_{j} ; \bar{x}^{i, j}\right)$ is the observed response for all the variables, $x_{k} \equiv \bar{x}_{k}, k \neq i, j$, fixed at the cut center except for $x_{i}$ and $x_{j}$. A similar interpretation would apply to higher order HDMR terms. In HDMRbased approximation, the notions of first-, second-order, etc. are used to define the constant term, terms with one variable or two variables only. It is recognized that the lower order (e.g., first-order or second-order) function expansions in the HDMR, do not generally translate to linear or quadratic functions. ${ }^{35}$ Each of the lower-order terms in the HDMR is sub-dimensional, but they are not necessarily low degree polynomials. The computational savings afforded by the HDMR are easily estimated. If the HDMR converges at $\beta$ order with acceptable accuracy and considering $s$ sample points for each variable, then the total number of numerical analyses needed to determine the $\operatorname{HDMR}$ is $\sum_{k=0}^{\beta}[M ! / k !(M-k) !](s-1)^{k}$.

\section{Numerical Results}

The effects of spatial uncertainty on the vibratory hub loads of a composite hingeless helicopter rotor are studied in this section. Uncertainty analysis is performed for two cases of rotors. In the first case, 
the uncertain stiffness properties are assumed to be similar for all of the four rotor blades which results in an isotropic rotor system. Therefore, the integer multiples of $4 / \mathrm{rev}$ vibratory loads are the only loads transmitted from blades to the fuselage. ${ }^{11}$ In the second case, uncertain stiffness properties of each of the four blades are considered to be independent from the other blades. Here, as discussed in the previous section, the uncertainty effects on $1 /$ rev to $5 /$ rev vibratory blade loads transmitted to the fuselage are studied.

The rotor blade is considered as a uniform blade equivalent of the BO-105 rotor blade and its properties are given in Table 1. The cross-section of rotor blade is modeled as a composite box beam with a breadth of $0.144 \mathrm{~m}$, and height of $0.081 \mathrm{~m}$ as shown in Fig. 1(b). All of the four walls of the box beam are considered to be made of a laminate with stacking sequence $\left[0_{3} / \pm 15_{3} / \pm 45_{2}\right]_{s}$. Each wall of the box beam is therefore made of a balanced symmetric laminate with 26 plies of graphite/epoxy material, where each ply is 0.127 $\mathrm{mm}$ thick. The box-beam type representation of the rotor blade is extensively used in rotorcraft aeroelastic analysis and optimization studies. ${ }^{13,36}$ Initially, a baseline analysis is carried out with the nominal material properties and the results of uncertainty analysis are compared with these baseline results.

Most studies on uncertainty analysis of composite structures consider $E_{1}, E_{2}, G_{12}$ and $\nu_{12}$ as statistically independent random variables. ${ }^{10}$ In this study, the composite material properties, $E_{1}, E_{2}, G_{12}$ and $\nu_{12}$, are considered as independent random variables with normal distributions. The COVs for each of the above material properties is taken from reference ${ }^{10}$ and listed in Table 2. Initially, the effect of uncertain material properties on the cross-sectional stiffnesses such as flap bending $\left(E I_{y}\right)$, lag bending $\left(E I_{z}\right)$ and torsional stiffness $(G J)$ of rotor blade are evaluated. With the statistical measures of uncertainty in crosssectional stiffness, the spatial variations of stiffness properties along the blade span are modeled. The stiffness properties are considered to be spatially varying along the axis of the blade $(\xi)$, as an independent, homogeneous, log-normal random fields. That is, the torsional stiffness $G J(\xi)$ is given as $c_{1} \exp \left[\alpha_{1}(\xi)\right]$, bending stiffnesses, $E I_{y}(\xi)$ as $c_{2} \exp \left[\alpha_{2}(\xi)\right]$ and $E I_{z}(\xi)$ as $c_{2} \exp \left[\alpha_{2}(\xi)\right]$. HDMR models are developed to approximate the aeroelastic outputs in terms of the blade stiffnesses as random fields. Uncertainty analysis of isotropic and dissimilar rotor systems are discussed in the following sections.

Table 1. Baseline hingeless rotor blade properties.

\begin{tabular}{ll}
\hline Number of blades & 4 \\
Radius, $R(\mathrm{~m})$ & 4.94 \\
Hover tip speed, $\Omega R(\mathrm{~m} / \mathrm{s})$ & 198.12 \\
Mass per unit length, $m_{o}(\mathrm{~kg} / \mathrm{m})$ & 6.46 \\
Lock number & 6.34 \\
Solidity & 0.10 \\
$C_{T} / \sigma$ & 0.07 \\
\hline
\end{tabular}

Table 2. Scatter in material properties.

\begin{tabular}{lll}
\hline Material properties & Mean & COV $(\%)$ \\
\hline$E_{1}(\mathrm{GPa})$ & 141.96 & 7.0 \\
$E_{2}(\mathrm{GPa})$ & 9.79 & 4.0 \\
$G_{12}(\mathrm{GPa})$ & 6.00 & 11.0 \\
$\nu_{12}$ & 0.42 & 4.0 \\
\hline
\end{tabular}

\section{A. Case I: Spatial Uncertainty with Similar Blades}

Uncertainty effects on the blade cross-sectional stiffnesses are evaluated with 5000 random samples of material properties with the COVs listed in Table $2 .{ }^{13}$ With these random material properties, the flap bending, lag bending and torsional stiffnesses of the rotor blade show COVs of 6.51,6.51, and 6.04 percent, respectively, as given in Table 3. The variation of the stiffness properties along the axis of rotor blade are now modeled with the above uncertainty results. The rotor blade is divided into 10 beam elements and the random fields 
Table 3. Statistics of cross-sectional stiffness values

\begin{tabular}{lcr}
\hline $\begin{array}{l}\text { Stiffness } \\
\text { (Non-dimensional) }\end{array}$ & Baseline value & $\begin{array}{r}\text { COV } \\
(\%)\end{array}$ \\
\hline$G J / m_{o} \Omega^{2} R^{4}$ & 0.003817 & 6.04 \\
$E I_{y} / m_{o} \Omega^{2} R^{4}$ & 0.008335 & 6.51 \\
$E I_{z} / m_{o} \Omega^{2} R^{4}$ & 0.023174 & 6.51 \\
\hline
\end{tabular}

are used to approximate the spatial variations. For a four bladed rotor considered in this study, the impact of spatial uncertainty on the $4 / \mathrm{rev}$ (per revolution) vibratory loads which are longitudinal shear $\left(F_{x}\right)$, lateral shear $\left(F_{y}\right)$, and vertical shear $\left(F_{z}\right)$ along with the rolling moment $\left(M_{x}\right)$, pitching moment $\left(M_{y}\right)$, and yawing moment $\left(M_{z}\right)$ are studied. ${ }^{22}$ The $4 /$ rev forces are normalized by the rotor steady thrust and the $4 / \mathrm{rev}$ moments are normalized by the rotor steady yawing moment. The baseline values of vibratory hub loads, at an advance ratio of 0.3 , are given in Fig. 2.
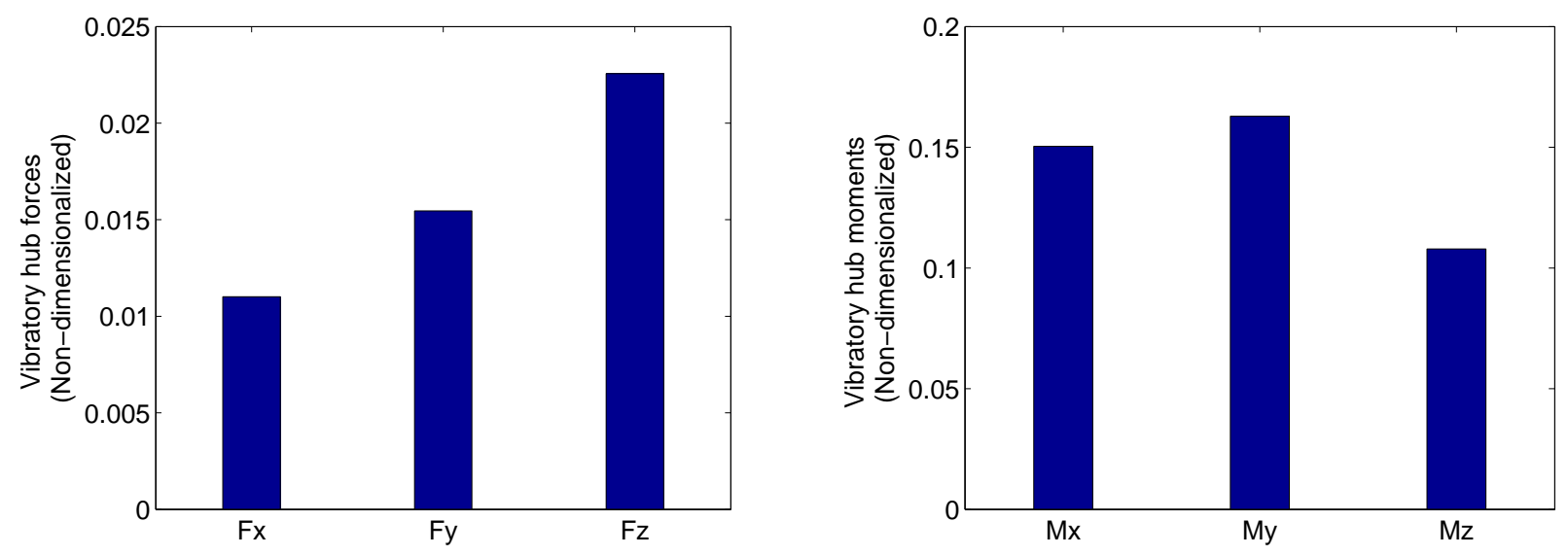

Figure 2. Baseline vibratory forces and moments.

\section{HDMR of Aeroelastic Model}

The HDMR models to approximate the aeroelastic response are discussed in this section. Based on the theory discussed in sections III and IV, the proposed methodology for the HDMR approximation is implemented using the following steps:

1. Represent the random field inputs in terms of standard random variables by a K-L expansion.

2. Select the reference point $\overline{\mathbf{x}}$, generally as the mean of the input variables.

3. Determine $f_{0}$, which is a constant term, representing the response at reference point $\overline{\mathbf{x}}$.

4. Generate regularly spaced sample points for each variable, as $x_{i}^{1}=\bar{x}_{i}-(s-1) k_{i} / 2, x_{i}^{2}=\bar{x}_{i}-$ $(s-3) k_{i} / 2, \ldots, x_{i}^{(s+1) / 2}=\bar{x}_{i}, \ldots, x_{i}^{s-1}=\bar{x}_{i}+(s-3) k_{i} / 2, x_{i}^{s}=\bar{x}_{i}+(s-1) k_{i} / 2$, along the variable axis $x_{i}$ with reference $\bar{x}_{i}$ and regular spacing $k_{i}$, which is the standard deviation of $x_{i}$. Here, $s$ denotes the number of HDMR sample points, and must be odd. More details about the choice of reference points and other details can be found in. ${ }^{33}$

5. Estimate the responses at the sample points for each variable using the computational model.

6. Construct the HDMR approximation using following steps: 
- Interpolate each of the low dimensional (e.g., first-order) HDMR expansion terms $f\left(x_{i} ; \bar{x}^{i}\right)$ as $f\left(x_{i} ; \bar{x}^{i}\right)=\sum_{j=1}^{s} \varphi_{j}\left(x_{i}\right) f\left(x_{i}^{j} ; \bar{x}^{i}\right)$. The response values are calculated in previous step and $\varphi_{j}\left(x_{i}\right)$ represents interpolation/shape functions. In this study, moving least squares interpolation functions are used and the details of which can be found in Ref. ${ }^{37}$

- Sum the interpolated values of HDMR expansion terms. This leads to the first-order HDMR approximation of the function $f(\mathbf{x})$ as follows:

$$
\hat{f}(\mathbf{x})=\sum_{1 \leq i \leq M} \sum_{j=1}^{s} \varphi_{j}\left(x_{i}\right) f\left(x_{i}^{j} ; \bar{x}^{i}\right)-(M-1) f(\overline{\mathbf{x}}) \quad \beta=1
$$

7. Perform Monte Carlo simulations using the approximated response function $\hat{f}(\mathbf{x})$.

8. Estimate the output statistics.

Numerical parameters of the above steps are discussed here. Normalized mean and COVs of the random fields, (i.e, flap bending, lag bending and torsional stiffness) are $\mu_{G J}=1.0, \mu_{E I_{y}}=1.0, \mu_{E I_{z}}=1.0$; and $v_{G J}=0.060, v_{E I_{y}}=0.065, v_{E I_{z}}=0.065$, respectively. $c_{1}=\mu_{G J} / \sqrt{1+v_{G J}^{2}}, c_{2}=\mu_{E I_{y, z}} / \sqrt{1+v_{E I_{y, z}}^{2}}$ and $\alpha_{1}(\xi), \alpha_{2}(\xi)$ are zero-mean, homogeneous, Gaussian random fields. The variance and covariance function of the random fields are $\sigma_{\alpha_{1}}^{2}=\ln \left(1+v_{G J}^{2}\right), \sigma_{\alpha_{2}}^{2}=\ln \left(1+v_{E I_{y, z}}^{2}\right)$ and $\Gamma_{\alpha_{1}}\left(\xi_{1}, \xi_{2}\right)=\sigma_{\alpha_{1}}^{2} \exp \left(-\left|\xi_{1}-\xi_{2}\right| / b\right)$, $\Gamma_{\alpha_{2}}\left(\xi_{1}, \xi_{2}\right)=\sigma_{\alpha_{2}}^{2} \exp \left(-\left|\xi_{1}-\xi_{2}\right| / b\right)$, respectively. $b$ is the correlation parameter that controls the rate at which the covariance decays. The degree of variability associated with the random process can be related to its coefficient of variation. The frequency content of the random field is related to the $L / b$ ratio in which $L$ is the length of the blade. $L / b=0.5$ is chosen in this study. In this example, the normalized length of the blade is $L=1$ and it is divided into 10 elements.

The eigensolutions of the covariance function are obtained by solving the integral equation (Eq. 7) analytically. The eigenvalues and eigenfunctions are given as follows:

$$
\begin{aligned}
\lambda_{i} & =\frac{2 \sigma_{\alpha}^{2} b}{\omega_{i}^{2}+b^{2}} \\
\lambda_{i}^{*} & =\frac{2 \sigma_{\alpha}^{2} b}{\omega_{i}^{* 2}+b^{2}} \\
\psi_{i}(\xi) & =\frac{\cos \left(\omega_{i} \xi\right)}{\sqrt{a+\frac{\sin \left(2 \omega_{i} \xi\right)}{2 \omega_{i}}}} \quad \text { for } i=\text { odd } \\
\psi_{i}(\xi) & =\frac{\sin \left(\omega_{i}^{*} \xi\right)}{\sqrt{a-\frac{\sin \left(2 \omega_{i}^{*} \xi\right)}{2 \omega_{i}^{*}}}} \quad \text { for } i=\text { even }
\end{aligned}
$$

The symbol $\omega_{i}$ presents the period of the random field. The random field is discretized into ten standard random variables, and the ten-term K-L expansion (Eq. (9)) is used to generate sample functions of the input random field. Realizations of the cross-sectional stiffnesses of the blade are numerically simulated using the K-L expansion method. The response quantities of the blade are represented by HDMR expansion with seven sample points. Thus, total number of function evaluations $((s-1) \times M+1=(7-1) \times 10+1)$ required for HDMR is 61 . It should be noted that the HDMR-based approach requires conditional responses at selected sample points and the sample points are chosen along each of the variable axis. It is found from the authors' previous studies that $\mathrm{s}=5$ or 7 works well for most of the problem. ${ }^{21,27,37}$ Therefore, results for $\mathrm{s}=7$ is used in this paper to construct HDMR-based metamodels.

\section{Uncertainty Analysis with HDMR models}

To evaluate the accuracy of the developed HDMR models, MCS with 1000 samples is performed with the HDMR models and the actual aeroelastic computational code, and the results are compared. Probability 
distribution functions (PDF) of the vibratory hub force $F_{z}$ and vibratory hub moment $M_{z}$ from the direct MCS and HDMR based MCS with 1000 samples are shown in Figs. 3. It is observed that the PDFs from MCS based on HDMR models are able to approximate, with reasonable accuracy, the PDFs from direct MCS.

The HDMR models are now used to evaluate the effects of the spatial uncertainty on the six vibratory hub loads with one hundred thousand samples. The PDFs of baseline vibratory forces and moments are shown in Fig. 4. The longitudinal vibratory force shows a deviation of -20 to 20 percent from its baseline value, the lateral force a deviation of -5 to 10 percent, and the vertical force a deviation of -60 to 40 percent. The vertical vibratory force is dominant among all three forces, as shown in Fig. 4, and is highly sensitive to the uncertainties. The rolling and pitching moments show a scattering of around -2 to 1 percent from their baseline values. However, the vibratory yawing moment shows scattering of around -20 to 60 percent. Further, the PDFs of 4/rev loads given in Fig. 4 show non-Gaussian type distributions. The results from this study show that parametric uncertainty plays a major role in reliable aeroelastic predictions and have to be incorporated in the design and optimization of composite aircraft structures. The CPU time for the aeroelastic simulations with 1000 samples is approximately $10 \mathrm{CPU}$ hours in a PC with a quad core processor. Therefore, for one hundred thousand samples, it would take approximately 1000 CPU hours which is very expensive compared to MCS with HDMR models that takes only a few minutes. Further, the HDMR models are developed with 61 aeroelastic simulations which take approximately 1 CPU hour only. Therefore, the development of approximation models such as HDMR methods can significantly reduce the computational cost of stochastic analysis.
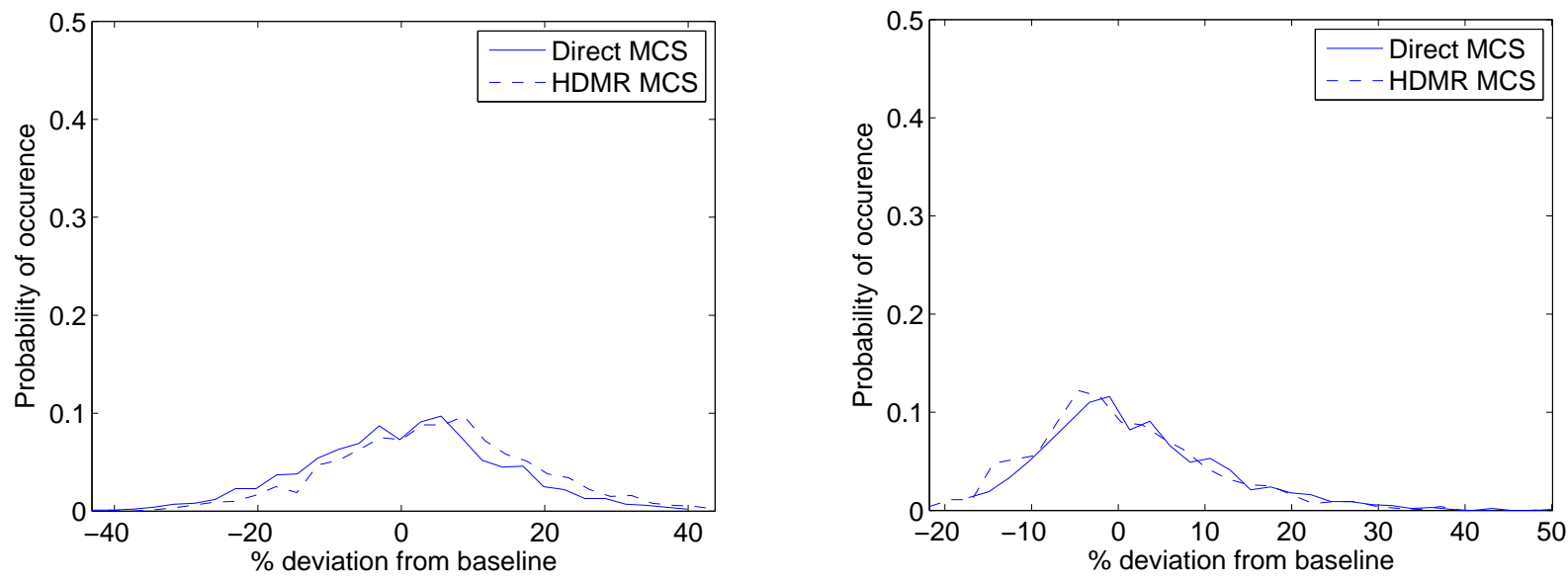

Figure 3. Comparison of the uncertainty results of direct MCS and HDMR based MCS of isotropic rotor system.

\section{B. Case II: Spatial Uncertainty with Dissimilar Blades}

In this section, the spatial uncertainty modeled on each rotor blade is different from the other blades. This results in a rotor with dissimilar blades which transmits all harmonics of vibratory loads acting on the blades to the fuselage. The spatial uncertainty of the dissimilar rotor with four blades is modeled with the following assumptions.

Case 1: Uncertainty assumed for one blade and the remaining blades are assumed to have its baseline values. Case 2: Uncertainty assumed for two blades which are diagonally opposite to each other.

Case 3: Uncertainty assumed for two consecutive blades.

Case 4: Uncertainty assumed for three blades.

Case 5: Uncertainty assumed for all four blades.

The effects of spatial uncertainty on the vibratory load predictions are different for each of the above assumption. A sensitivity analysis is performed for each of the above assumed conditions. Further, the magnitude of the effects of the uncertain flap, lag and torsional stiffness can be different from each other. 
This sensitivity analysis can be used to find the highly sensitive uncertain variables and reduce the number of design variables used in the surrogate models. The flap, lag and torsion stiffness of rotor blades are assumed to be spatially uncertain with the COVs given in Table 3 . The effects of the spatial uncertainty on the $1 / \mathrm{rev}$ to $5 / \mathrm{rev}$ vibratory loads transmitted to the fuselage for cases 1 to 5 are given in Figs. 5 to 7 . The vibratory loads of $1 / \mathrm{rev}, 2 / \mathrm{rev}, 3 / \mathrm{rev}$ and $5 / \mathrm{rev}$ are non-dimensionalized with their corresponding $4 / \mathrm{rev}$ loads. This study shows that the uncertain dissimilar rotor system can induce $1 /$ rev and $2 /$ rev vibratory hub forces with magnitudes of $10-60$ percent of the $4 /$ rev loads. Further, the vibratory loads are highly sensitive to the uncertainties in lag and torsional stiffness compared to the flap stiffness. Figure 8 shows that the uncertainty in flap, lag and torsional stiffnesses of all four blades can significantly induce the $1 / \mathrm{rev}$ and $2 /$ rev vibratory forces as comparable to $4 /$ rev forces. Further, these study shows that the uncertainty effects mainly depend on the type of blade stiffness (flap, lag or torsion) rather than the number of uncertain blades. However, these sensitivity analysis results are based on the simulation with only one random sample for each of the above cases considered.

An uncertainty analysis based on Monte Carlo simulations is performed to identify the $n /$ rev vibratory hub forces and moments which are highly sensitive to the uncertainty. Monte Carlo simulations of the dissimilar rotor is performed with 100 samples with uncertain flap, lag and torsional stiffness of all four blades. The MCS is performed with the COVs of flap, lag and torsional stiffness as $6.51,6.51$ and 3.5 percent and the results are shown in Figs. 9 and 10. The results show that the $1 / \mathrm{rev}$ and $2 / \mathrm{rev}$ forces of $F x$ and $F y$, and $1 /$ rev and $4 /$ rev forces of $F z$ are highly sensitive to the uncertainty. Similarly, the $1 /$ rev vibratory moments of $M x, M y$, and $M z$, and 4/rev of $M z$ are highly sensitive to the spatially uncertain rotor blades. HDMR models of these specified vibratory forces and moments can be developed as discussed in the previous sections and this work is in progress. HDMR models can be used to perform MCS with sufficient samples to obtain the probability distributions of the vibratory loads of dissimilar rotor systems.

\section{Conclusions}

The effects of spatially uncertain flap, lag and torsional stiffness of blade properties on the vibratory hub loads has been studied. The spatial uncertainty analysis is performed with rotors modeled as isotropic rotors and dissimilar rotors. In the isotropic rotor system, the uncertainty is considered to be same for all the four blades and the uncertainty effects on the 4/rev vibratory hub loads are studied. A high dimensional model representation of the computational aeroelastic model is developed to reduce the computational cost of uncertainty analysis, and Monte Carlo simulations are then performed with these developed models. Uncertainty analysis of the dissimilar rotor system is studied with each of the rotor blades possessing different spatially uncertain stiffness properties. The effects of spatially uncertain blades of the dissimilar rotor on the $1 /$ rev to $5 /$ rev vibratory hub loads are studied with MCS. The following conclusions are drawn from this study:

1. Spatial uncertainty of the rotor with similar blades have considerable influence on the $4 /$ rev vibratory hub loads of the helicopter. Longitudinal and lateral vibratory forces show deviations up to 20 percent from their baseline value. The vertical vibratory force is highly sensitive to uncertainty with maximum deviations up to 60 percent from its baseline value. The rolling and pitching moments are less sensitive to uncertainty whereas the vibratory yawing moment shows a scattering of up to 60 percent. The PDFs of 4/rev vibratory hub loads loads show non-Gaussian type distributions.

2. High dimensional model representations of the response are found to approximate the computational aeroelastic model for uncertainty analysis. Monte Carlo simulations with the HDMR models and direct simulations produce similar results. Therefore, Monte carlo simulations with a very large number of samples (one hundred thousand) are performed with HDMR models in few minutes which can take approximately $1000 \mathrm{CPU}$ hours with the direct simulations. Further, it is found that HDMR models with $s=7$ are found to produce good meta-model approximations.

3. Spatial uncertainty of rotors with dissimilar blades have considerable influence on the $1 /$ rev and $2 /$ rev vibratory hub loads in addition to the $4 /$ rev vibratory hub loads. The $1 /$ rev and $2 /$ rev harmonics of longitudinal and lateral vibratory hub forces, and the $1 /$ rev vertical vibratory hub force are highly sensitive to spatial uncertainties. Similarly, the $1 /$ rev harmonics of the rolling, pitching and yawing vibratory hub moments are highly sensitive to spatial uncertainties of the rotor blades. 


\section{Acknowledgments}

The authors acknowledge the support of the European Research Council through project 247045 entitled "Optimisation of Multi-scale Structures with Applications to Morphing Aircraft".

\section{References}

${ }^{1}$ Pettit, C. L., "Uncertainty quantification in aeroelasticity: Recent results and research challenges," Journal of Aircraft, Vol. 41, No. 5, 2004, pp. 1217-1229.

${ }^{2}$ Poirion, F., "On some stochastic methods applied to aeroservoelasticity," Aerospace Science and Technology, Vol. 4, No. 3, 2000, pp. $201-214$.

${ }^{3}$ Lindsley, N. J., Beran, P. S., and Pettit, C. L., "Effects of uncertainty on nonlinear plate aeroelastic response," AIAA Paper 2002-1271, 2002.

${ }^{4} \mathrm{Wu}, \mathrm{C}$., Zhang, H., and Fang, T., "Flutter analysis of an airfoil with bounded random parameters in incompressible flow via Gegenbauer polynomial approximation," Aerospace Science and Technology, Vol. 11, No. 7-8, 2007, pp. 518 - 526.

${ }^{5}$ Bruno, L., Canuto, C., and Fransos, D., "Stochastic aerodynamics and aeroelasticity of a flat plate via generalised Polynomial Chaos," Journal of Fluids and Structures, Vol. 25, No. 7, 2009, pp. 1158 - 1176.

${ }^{6}$ Borello, F., Cestino, E., and Frulla, G., "Structural uncertainty effect on classical wing flutter characteristics," Journal of Aerospace Engineering, Vol. 23, No. 4, 2010, pp. 327-338.

${ }^{7}$ Marques, S., Badcock, K. J., Haddad Khodaparast, H., and Mottershead, J. E., "Transonic aeroelastic stability predictions under the influence of structural variability," Journal of Aircraft, Vol. 47, No. 4, 2010, pp. 1229-1239.

${ }^{8}$ Ghommem, M., Hajj, M., and Nayfeh, A., "Uncertainty analysis near bifurcation of an aeroelastic system," Journal of Sound and Vibration, Vol. 329, No. 16, 2010, pp. 3335 - 3347.

${ }^{9}$ Sriramula, S. and Chryssanthopoulos, M. K., "Quantification of uncertainty modelling in stochastic analysis of FRP composites," Composites Part A: Applied Science and Manufacturing, Vol. 40, No. 11, 2009, pp. 1673 - 1684.

${ }^{10}$ Onkar, A. K., Upadhyay, C. S., and Yadav, D., "Stochastic finite element buckling analysis of laminated plates with circular cutout under uniaxial compression," Journal of Applied Mechanics-Transactions of the Asme, Vol. 74, No. 4, 2007, pp. 798-809.

${ }^{11}$ Johnson, W., Helicopter Theory, Princeton University Press, New Jersy, 1980.

${ }^{12}$ Murugan, S., Harursampath, D., and Ganguli, R., "Material uncertainty propagation in helicopter nonlinear aeroelastic response and vibration analysis," AIAA Journal, Vol. 46, No. 9, 2008, pp. 2332-2344.

${ }^{13}$ Murugan, S., Ganguli, R., and Harursampath, D., "Aeroelastic response of composite helicopter rotor with random material properties," Journal of Aircraft, Vol. 45, No. 1, 2008, pp. 306-322.

${ }^{14}$ Siva, C., Murugan, M. S., and Ganguli, R., "Effect of uncertainty on helicopter performance predictions," Proceedings of the Institution of Mechanical Engineers, Part G: Journal of Aerospace Engineering, Vol. 224, No. 5, 2010, pp. 549-562.

${ }^{15}$ Murugan, S., Ganguli, R., and Harursampath, D. K., "Stochastic aeroelastic analysis of composite helicopter rotor," Journal of the American Helicopter Society, Vol. 56, No. 1, 2011, pp. 012001.

${ }^{16}$ You, Y. H., Jung, S. N., Pawar, P. A., and Shin, E. S., "Effect of uncertainty on hub vibration response of composite helicopter rotor blades," Journal of Aircraft, Vol. 47, No. 1, JAN-FEB 2010, pp. 151-160.

${ }^{17}$ Attar, P. J. and Dowell, E. H., "Stochastic analysis of a nonlinear aeroelastic model using the response surface method," Journal of Aircraft, Vol. 43, No. 4, 2006, pp. 1044-1052.

${ }^{18}$ Vanmarcke, E., Random Fields : Analysis and Synthesis, The MIT Press, Cambridge, Massachussets, 1993.

${ }^{19}$ Rabitz, H. and Alis, O., "General foundations of high-dimensional model representations," Journal of Mathematical Chemistry, Vol. 25, No. 2-3, 1999, pp. 197-233.

${ }^{20} \mathrm{Li}$, G., Wang, S., Rabitz, H., Wang, S., and Jaffe, P., "Global uncertainty assessments by high dimensional model representations (HDMR)," Chemical Engineering Science, Vol. 57, No. 21, 2002, pp. 4445-4460.

${ }^{21}$ Chowdhury, R. and Rao, B. N., "Assessment of high dimensional model representation techniques for reliability analysis," Probabilistic Engineering Mechanics, Vol. 24, No. 1, 2009, pp. 100-115.

${ }^{22}$ Bir, G. and Chopra, I., "University of Maryland Advanced Rotorcraft Code (UMARC) Theory Manual," Tech. Rep. UM-AERO Report 92-02, 1992.

${ }^{23}$ Viswamurthy, S. R. and Ganguli, R., "An optimization approach to vibration reduction in helicopter rotors with multiple active trailing edge flaps," Aerospace Science and Technology, Vol. 8, No. 3, 2004, pp. 185 - 194.

${ }^{24}$ Ganguli, R., "A survey of recent developments in rotorcraft design optimization," Journal of Aircraft, Vol. 41, No. 3, 2004, pp. 493-510.

${ }^{25}$ Roget, B. and Chopra, I., "Individual blade control methodology for a rotor with dissimilar blades," Journal of the American Helicopter Society, Vol. 48, No. 3, 2003, pp. 176-185.

${ }^{26}$ Sudret, B. and Der-Kiureghian, A., "Stochastic Finite Element Methods and Reliability," Tech. Rep. UCB/SEMM2000/08, Department of Civil \& Environmental Engineering, University Of California, Berkeley, November 2000.

${ }^{27}$ Chowdhury, R. and Adhikari, S., "High dimensional model representation for stochastic finite element analysis," Applied Mathematical Modelling, Vol. 34, No. 12, 2010, pp. 3917-3932.

${ }^{28}$ Nouy, A., "Recent developments in spectral stochastic methods for the numerical solution of stochastic partial differential equations," Archives of Computational Methods in Engineering, Vol. 16, No. 3, 2009, pp. 251-285.

${ }^{29}$ Babuska, I., Liu, K., and Tempone, R., "Solving stochastic partial differential equations based on the experimental data," Mathematical Models \& Methods in Applied Sciences, Vol. 13, No. 3, 2003, pp. 415-444. 
${ }^{30}$ Nouy, A., "Generalized spectral decomposition method for solving stochastic finite element equations: Invariant subspace problem and dedicated algorithms," Computer Methods in Applied Mechanics and Engineering, Vol. 197, No. 51-52, 2008, pp. $4718-4736$.

${ }^{31}$ Babuka, I. and Chatzipantelidis, P., "On solving linear elliptic stochastic partial differential equations," Computer Methods in Applied Mechanics and Engineering, Vol. 191, No. 37-38, 2002, pp. 4093-4122.

${ }^{32} \mathrm{Li}$, G., Rosenthal, C., and Rabitz, H., "High dimensional model representations," Journal of Physical Chemistry A, Vol. 105, No. 33, 2001, pp. 7765-7777.

${ }^{33}$ Rao, B. N. and Chowdhury, R., "Enhanced high-dimensional model representation for reliability analysis," International Journal for Numerical Methods in Engineering, Vol. 77, No. 5, 2009, pp. 719-750.

${ }^{34}$ Sobol, I., "Theorems and examples on high dimensional model representation," Reliability Engineering 85 System Safety, Vol. 79, No. 2, 2003, pp. 187-193.

${ }^{35}$ Chowdhury, R., Rao, B. N., and Prasad, A. M., "High-dimensional model representation for structural reliability analysis," Communications in Numerical Methods in Engineering, Vol. 25, No. 4, 2009, pp. 301-337.

${ }^{36}$ Soykasap, O., "Inverse method in tilt-rotor optimization," Aerospace Science and Technology, Vol. 5, No. 7, 2001, pp. 437 -444 .

${ }^{37}$ Chowdhury, R., Rao, B. N., and Prasad, A. M., "High dimensional model representation for piece-wise continuous function approximation," Communications in Numerical Methods in Engineering, Vol. 24, No. 12, Sp. Iss. SI, 2008, pp. 1587-1609. 


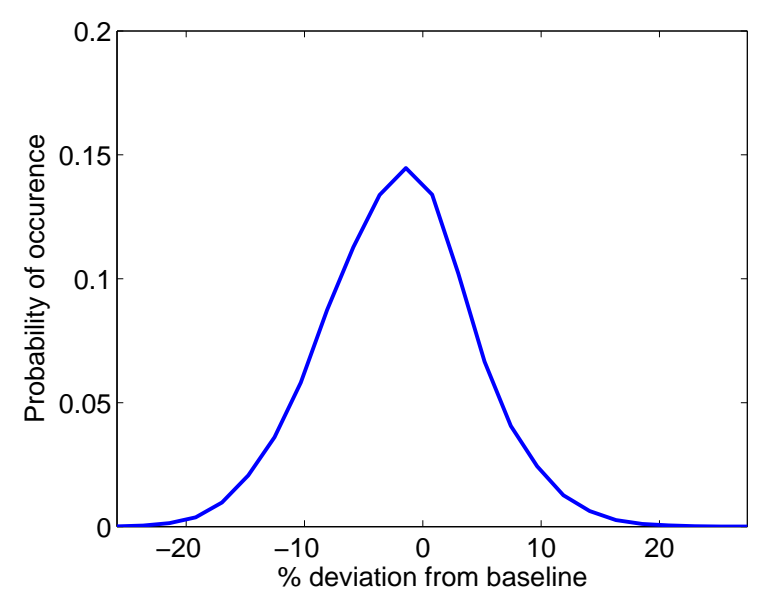

Vibratory force, Fx

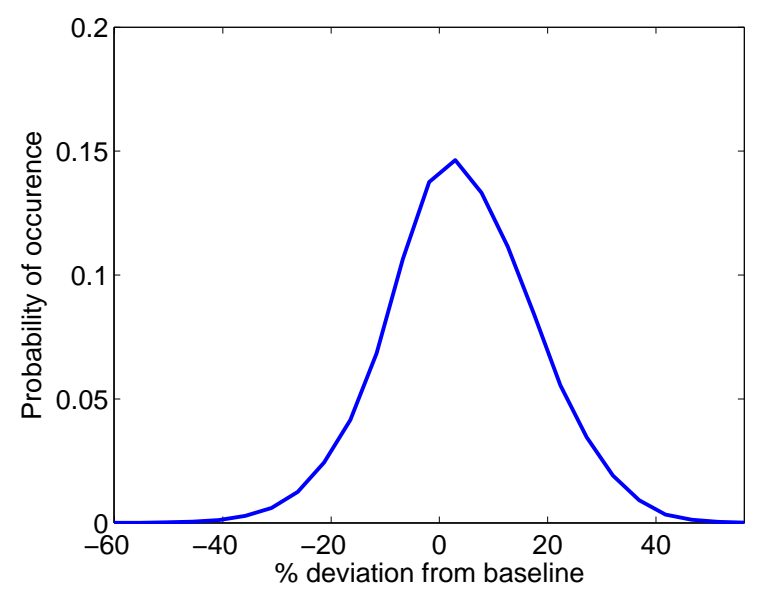

Vibratory force, $\mathrm{Fz}$

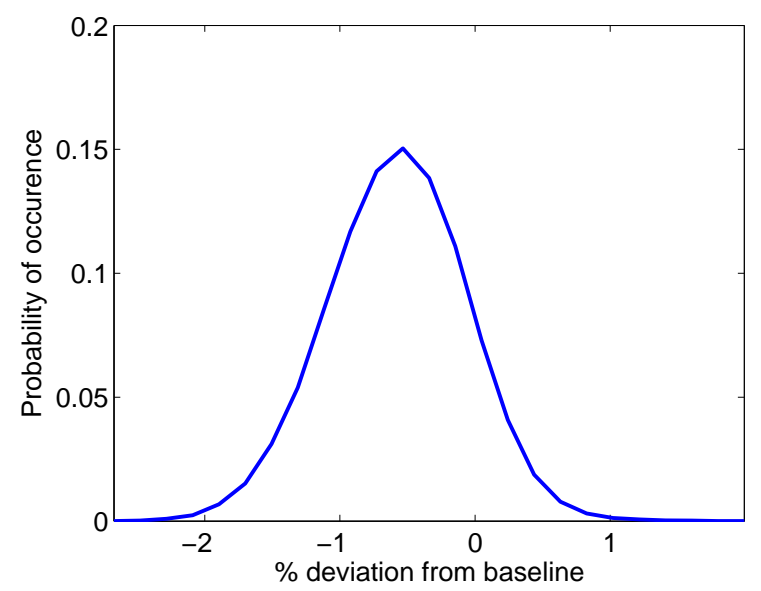

Vibratory moment, My

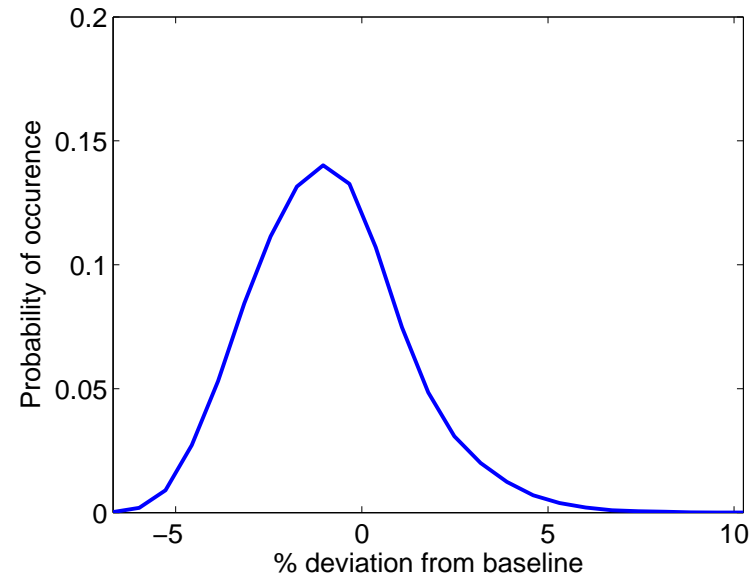

Vibratory force, Fy

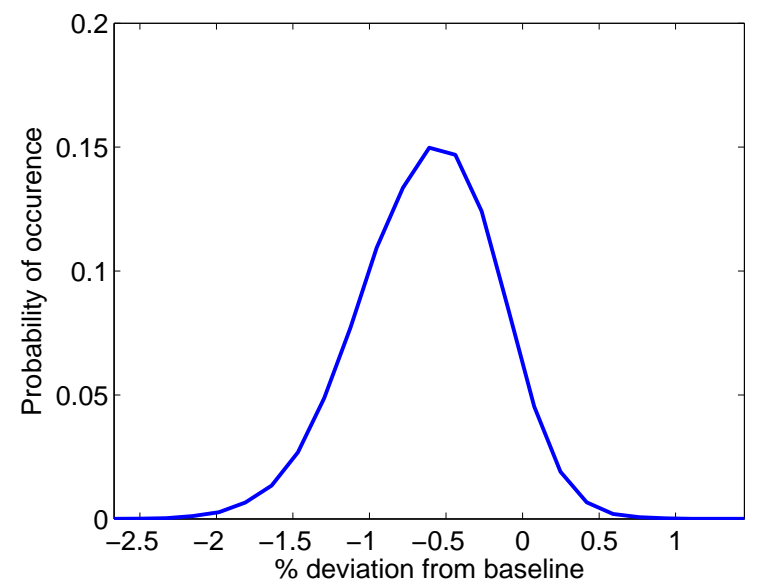

Vibratory moment, $\mathrm{Mx}$

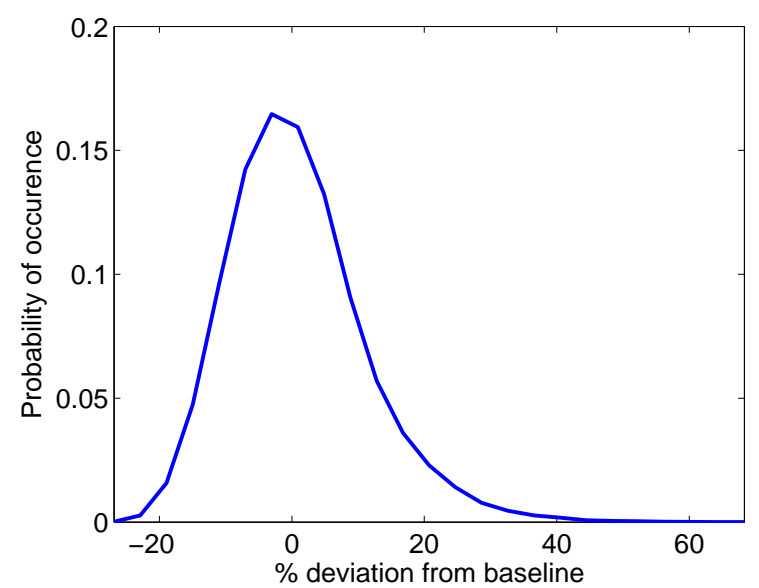

Vibratory moment, $\mathrm{Mz}$

Figure 4. Effects of spatial uncertainty on 4/rev vibratory loads of isotropic rotor system with HDMR based MCS. 

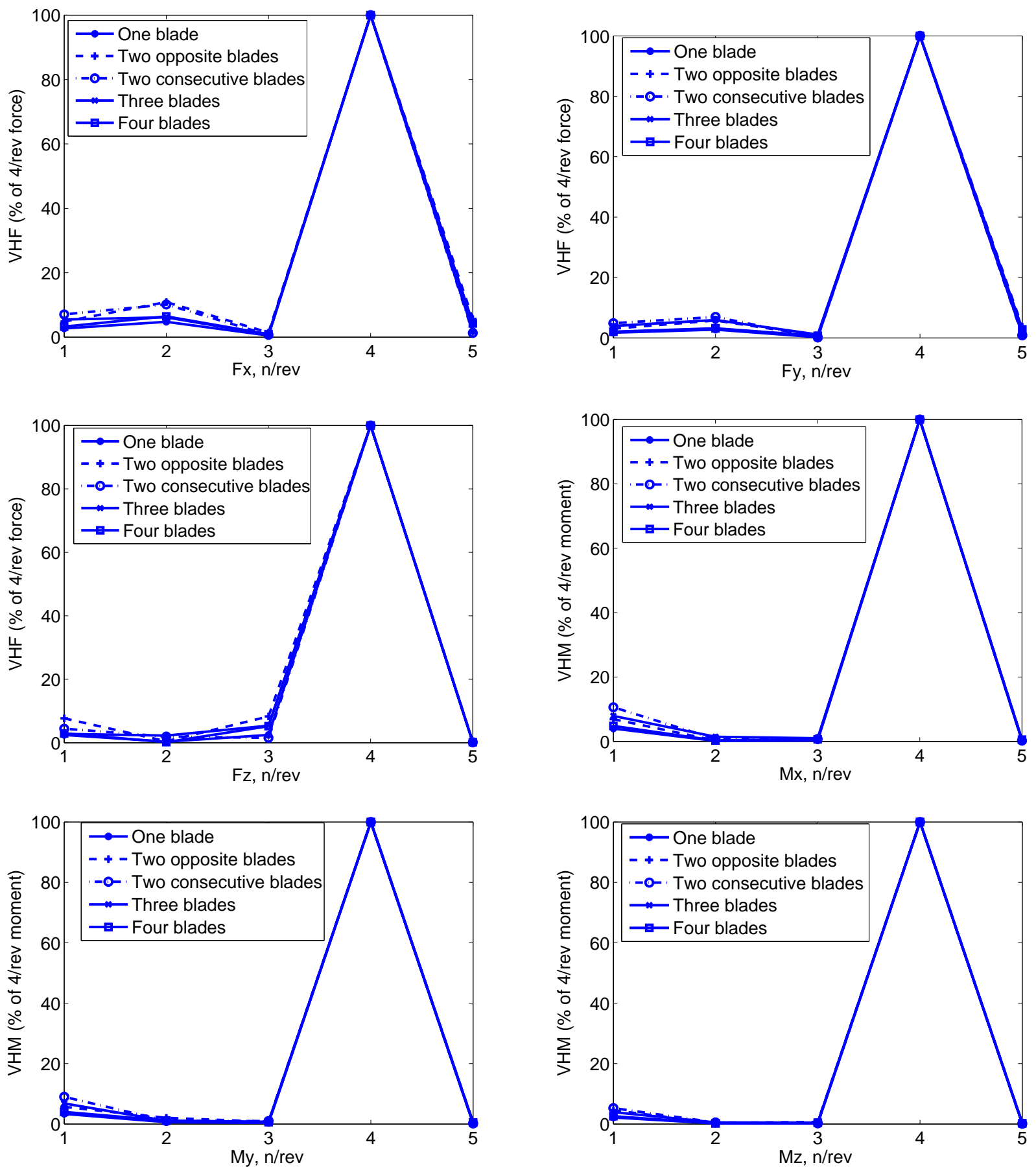

Figure 5. Effects of spatially uncertain flap stiffness on the vibratory hub forces (VHF) and moments (VHM) of dissimilar rotor blades. 

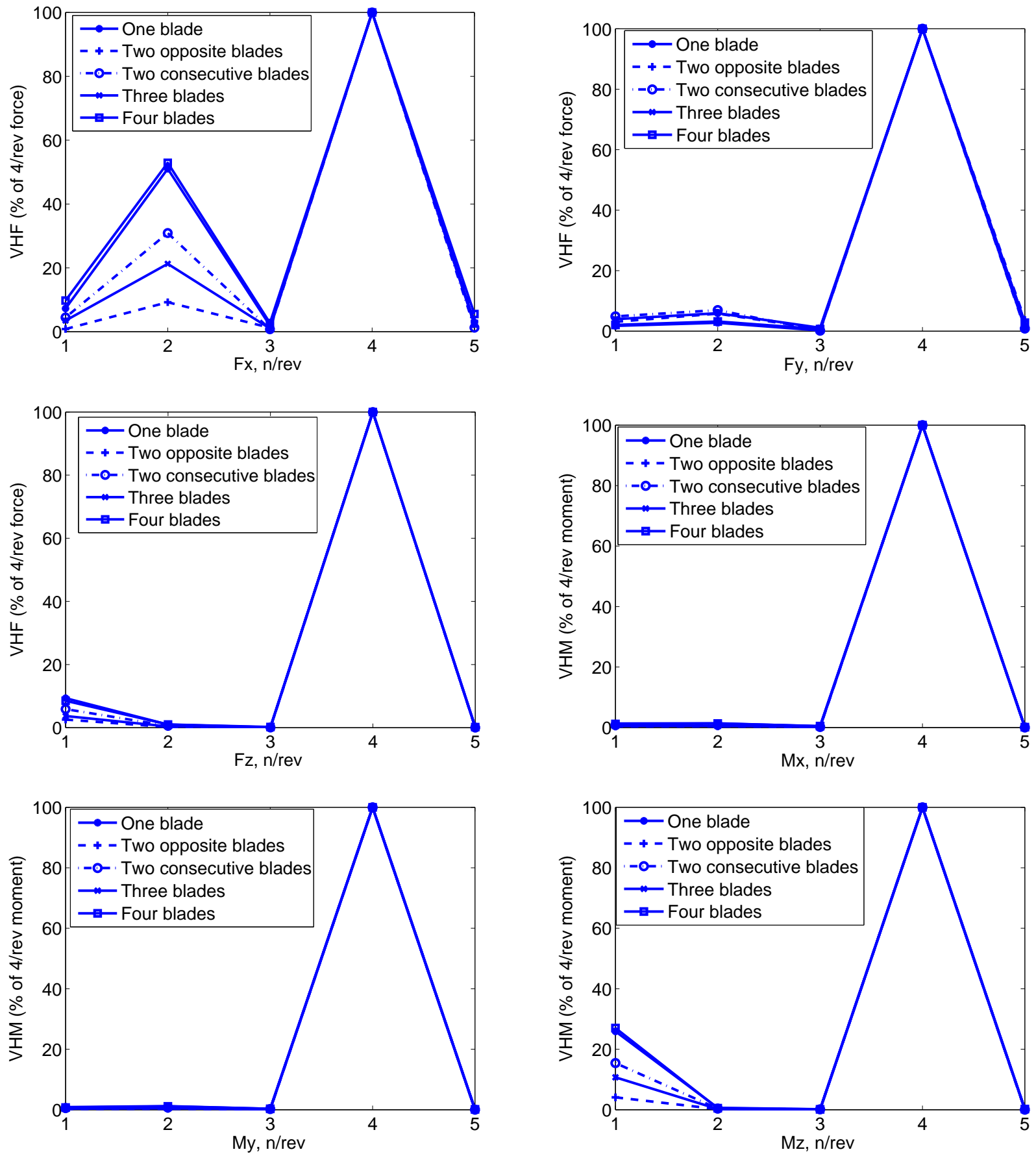

Figure 6. Effects of spatially uncertain lag stiffness on the vibratory hub forces (VHF) and moments (VHM) of dissimilar rotor blades. 

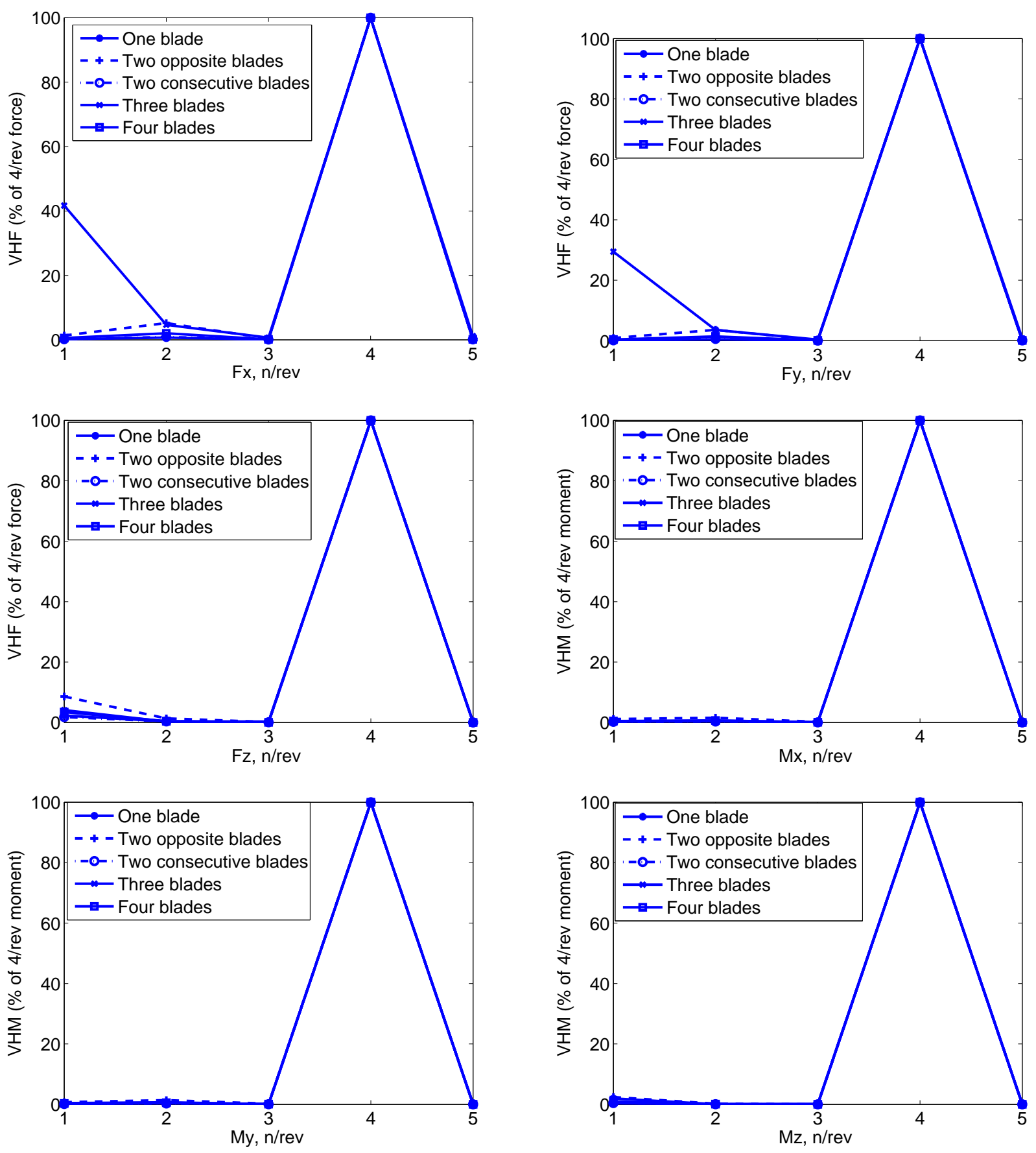

Figure 7. Effects of spatially uncertain torsional stiffness on the vibratory hub forces (VHF) and moments (VHM) of dissimilar rotor blades. 

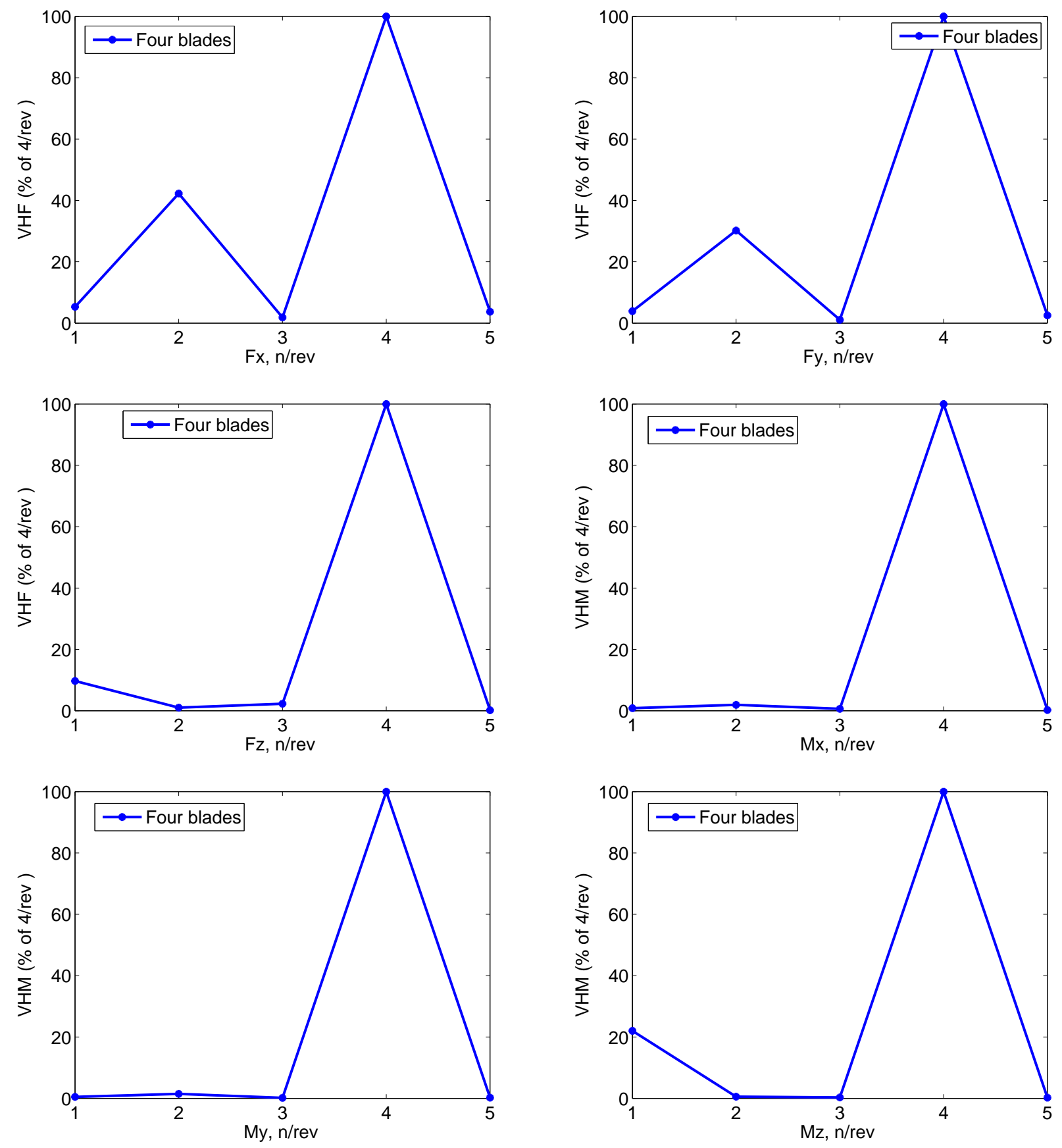

Figure 8. Effects of spatially uncertain stiffnesses (flap, lag or torsion) on the vibratory hub forces (VHF) and moments (VHM) of dissimilar rotor blades. 

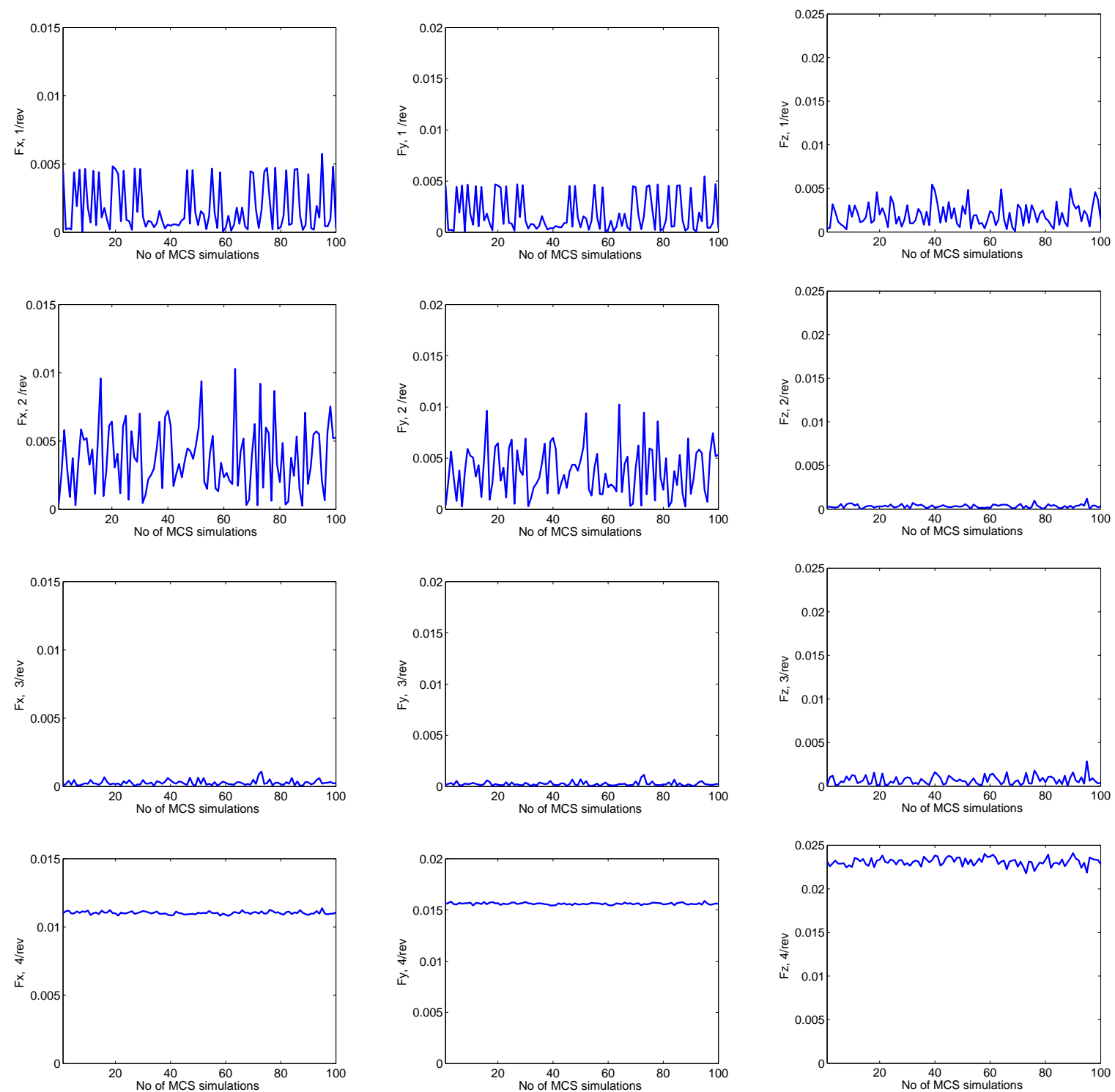

Figure 9. MCS of $1 / \mathrm{rev}, 2 / \mathrm{rev}, 3 / \mathrm{rev}$, and 4/rev vibratory hub forces $(F x, F y, F z)$ of spatially uncertain dissimilar rotor blades. 

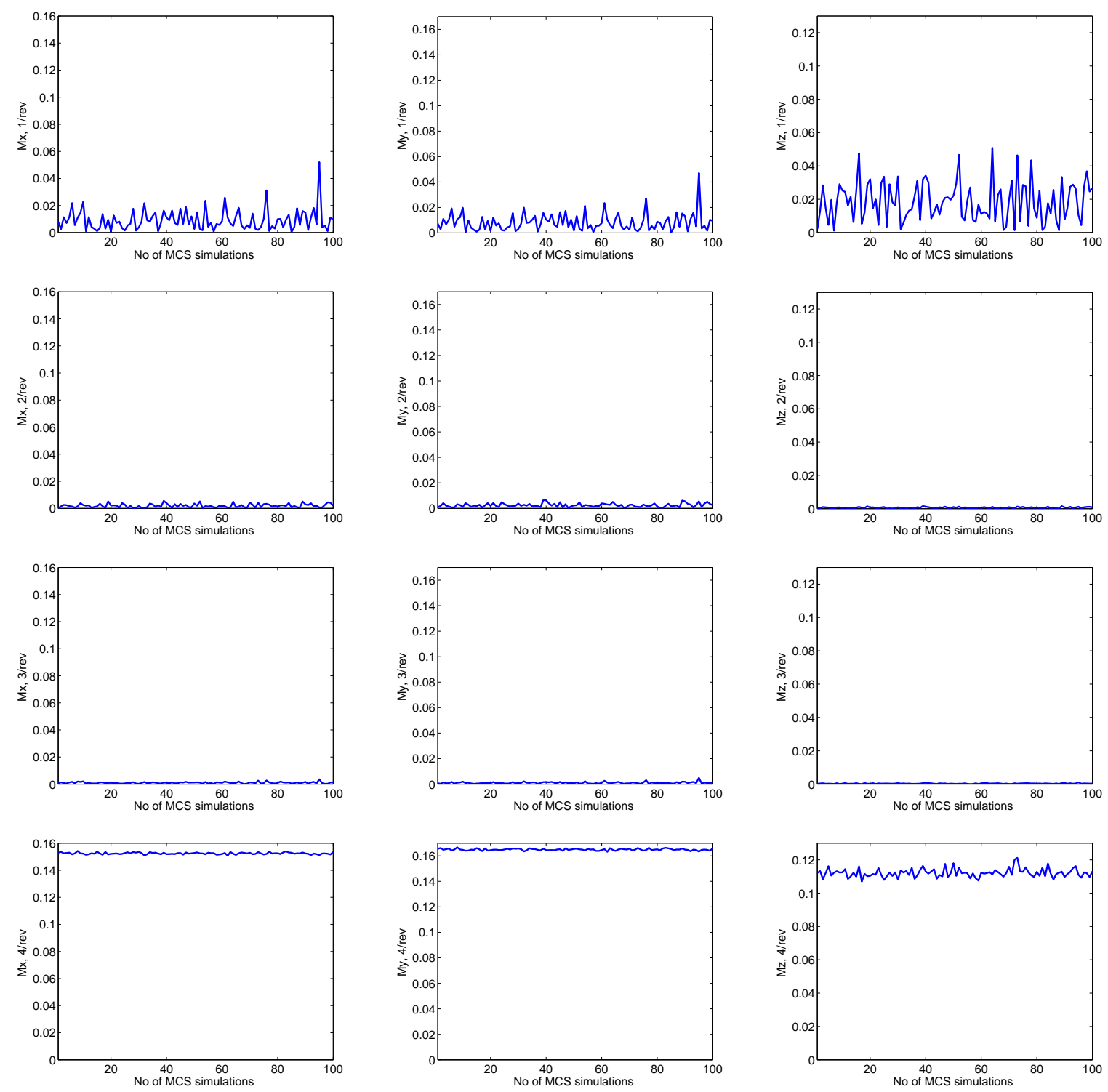

Figure 10. MCS of $1 / \mathrm{rev}, 2 / \mathrm{rev}, 3 / \mathrm{rev}$, and 4/rev vibratory hub moments $(M x, M y, M z)$ of spatially uncertain dissimilar rotor blades. 